\title{
Efficient Profile-Likelihood Confidence Intervals for Capture-Recapture Models
}

\author{
Olivier Gimenez, Rémi Choquet, Laurent LAMOR, Paul Scofield, \\ David FleTCHER, Jean-Dominique LEBRETON, and Roger PRADEL
}

In a capture-recapture analysis, uncertainty in the parameter estimates is usually expressed by presenting classical Wald-type confidence intervals. This approach involves (1) the assumption that the maximum likelihood estimates are asymptotically normal and (2) numerical computation of the variance-covariance matrix of these estimates. When the sample size is small or when the estimates are on the boundary of their domain, a Wald confidence interval often performs badly. A natural alternative is to use profile-likelihood confidence intervals. In general, these intervals require a greater amount of computation. We propose a new implementation of this approach that is efficient, both in reducing the amount of computation and in coping with boundary estimates. We also show how profilelikelihood confidence intervals can be adjusted for overdispersion. Simulations were used to check whether nominal coverage levels were attained, and allowed us to compare this approach with the classical Wald procedure. We illustrate this work by considering a multistate model for a sooty shearwater (Puffinus griseus) population.

Key Words: Boundary estimate; Multi-state model; Nominal coverage level; Overdispersion; Profile-likelihood interval; Venzon and Moolgavkar's algorithm.

\section{INTRODUCTION}

Multi-state capture-recapture models (Arnason 1972, 1973; Schwarz, Schweigert, and Arnason 1993; Lebreton and Pradel 2002; Williams, Nichols, and Conroy 2002, pp. 454468) are a natural generalization of the single-state Cormack-Jolly-Seber (CJS) model

Olivier Gimenez is TKKK, Centre d'Ecologie Fonctionnelle et Evolutive, Centre National de la Recherche Scientifique, Equipe Biométrie et Biologie des Populations, 1919 Route de Mende - 34293 Montpellier Cedex 5, France and Institut de l'Ingénierie de l'Information de Santé Laboratoire TIMC UMR CNRS 5525, équipe TIMB Faculté de Médecine - 38706 La Tronche Cedex, France (E-mail: olivier.gimenez@cefe.cnrs.fr). Rémi Choquet is TKKK, and Laurent Lamor is TKKK, Centre d'Ecologie Fonctionnelle et Evolutive, Centre National de la Recherche Scientifique, Equipe Biométrie et Biologie des Populations, 1919 Route de Mende - 34293 Montpellier Cedex 5, France. Paul Scofield is TKKKK, Canterbury Museum, Rolleston Avenue, Christchurch, 8001 New Zealand. David Fletcher is TKKK, Department of Mathematics and Statistics, University of Otago, Box 56, Dunedin, New Zealand. Jean-Dominique Lebreton is TKKKK, and Roger Pradel is TKKKK, Centre d'Ecologie Fonctionnelle et Evolutive, Centre National de la Recherche Scientifique, Equipe Biométrie et Biologie des Populations, 1919 Route de Mende - 34293 Montpellier Cedex 5, France

(C)2005 American Statistical Association and the International Biometric Society

Journal of Agricultural, Biological, and Environmental Statistics, Volume 10, Number 2, Pages 1-13

DOI: $10.1198 / 108571105 X 46462$ 
(Cormack 1964; Jolly 1965; Seber 1965) for the estimation of survival probabilities using captures or resightings of marked individuals (Lebreton, Almeras, and Pradel 1999). In a multi-state model, an individual can move between states, with extra parameters representing the probabilities of such transitions. States may correspond to geographical sites (multi-site models) or to states defined at the individual level, such as reproductive state. These models provide a great deal of flexibility in both modeling biological phenomena (Clobert 1995; Nichols and Kendall 1995; Lebreton and Pradel 2002) and in addressing a variety of biologically relevant questions (Lebreton and Pradel 2002; Williams et al. 2002, pp. 454-468).

Both special cases and generalizations of the basic time-dependent multi-state model have been proposed (Brownie et al. 1993). Lebreton et al. (2003), Lebreton and Pradel (2002), and Williams et al. (2002, pp. 454-468) described recent generalizations, in particular age-dependent multi-state models that allow estimation of both recruitment and age-dependent dispersal probabilities.

These developments have been made possible largely by the development of appropriate software (MARK, White and Burnham 1999; MSSURVIV, Hines 1994; M-SURGE, Choquet et al. 2003). In these programs, maximum likelihood estimates (MLE) of the parameters $(\theta)$ are obtained numerically (Brownie et al. 1993) and confidence intervals can be calculated using a variety of methods.

The most common method for calculating confidence intervals is the so-called Wald procedure, which relies on the asymptotic normality of $\hat{\theta}$, the MLE of $\theta$. However, Wald-type intervals can perform very badly for small sample sizes due to poor estimates of sampling variance, bias in the MLE, and/or asymmetry in the sampling distribution of the MLE (Beale 1960; Hudson 1971; Donaldson and Schnabel 1987). These problems are known to occur for both open (single-state) and closed capture-recapture models (White, Anderson, Burnham, and Otist 1982; Garthwaite and Buckland 1992; Evans, Kim, and O'Brien 1996). Although, to our knowledge, no empirical study of such intervals has been carried out for multi-state capture-recapture models, the complexity of the likelihood for such models and the often large number of parameters they involve (Viallefont and Lebreton 1993) suggest that these problems can only worsen. In particular, Ratkowsky (1988, pp. 20-25) argued that in general Wald-type intervals can be a poor choice in the context of nonlinear modeling (see also Cook and Weisberg 1990).

Burnham et al. (1987 p. 214) advocated transformation of $\theta$ onto the logistic scale [implemented in software MARK (White and Burnham 1999) and M-SURGE (Choquet et al. 2003)] in order to obtain improved confidence intervals, followed by back-transformation to the original scale (Lebreton, Burnham, Clobert, and Anderson 1992). However, as pointed out by Buckland and Garthwaite (1991), such a generic transformation may not be efficient for all situations. Moreover, when dealing with probabilities, use of a logistic transformation can often lead to any boundary estimates having an estimated variance effectively equal to zero.

The alternative of using profile-likelihood confidence intervals (Cox and Hinkley 1974, p. 343) therefore has some appeal (Lebreton 1995; Seber and Schwarz 2002). These intervals 
are generally expected to provide coverage rates closer to nominal levels than Wald-type intervals (Ratkowsky 1988, pp. 20-25; Cook and Weisberg 1990), even for small sample sizes (Bates and Watts 1980). Cormack (1992), Agresti (1994), Lloyd (1995), and Evans et al. (1996) made use of profile-based confidence intervals for estimating the size of an animal population, while Morgan and Freeman (1989) derived profile-based confidence intervals for the survival probabilities of first-year birds with ring-recovery data and Lebreton et al. (1992) provided an example for the CJS model.

Calculation of a profile-likelihood interval requires time-consuming optimization over the other parameters. To help overcome this problem, Venzon and Moolgavkar (1988) proposed an algorithm that requires less computation. We therefore consider it worthwhile investigating the performance of profile-likelihood-based confidence intervals for open multi-state capture-recapture models, and comparing these with Wald-type intervals [note that the usual CJS model is a particular case of a multi-state model (Lebreton et al. 1999)].

In the following section we apply the procedure proposed by Venzon and Moolgavkar (1988) to multi-state capture-recapture models. We then conduct a Monte Carlo study of the performances of both profile-likelihood and Wald-type intervals for such models. Bootstrap-based confidence intervals (Efron and Tibshirani 1993; Davison and Hinkley 1997) could also have been investigated in this article. In a capture-recapture context, most of the work on bootstrap intervals has focused on estimation of population size (Huggins 1989; Garthwaite and Buckland 1992; Evans et al. 1996), while use of such intervals for open single-state capture-recapture models has received little attention (Buckland 1980; Buckland and Garthwaite 1991); for multi-state models, there appears to be no published work in this area. The bootstrap approach is currently not implemented in standard capturerecapture software, probably due to the computational burden. We therefore decided not to consider bootstrap intervals in our simulations, although they may provide a good alternative to profile-likelihood intervals. Finally, we compare the performance of Wald, bootstrap, and profile-likelihood intervals for a set of multi-state capture-recapture data from a population of sooty shearwater (Puffinus griseus).

\section{PROFILE-LIKELIHOOD BASED CONFIDENCE INTERVALS FOR CAPTURE-RECAPTURE MODELS}

\subsection{Comparison With Traditional Approaches}

This work was motivated by estimation problems encountered while analyzing the transitions between reproductive states (breeder and non-breeder) of Sooty shearwaters (Puffinus griseus). The dataset encompasses seven years (Table 1) and was initially analyzed by Scofield, Fletcher, and Robertson (2001).

The model we considered is denoted $\left\{F_{f}, P_{c}, \Psi_{\text {f.to.t }}\right\}$ because it involves:

- survival probabilities $F$ that depend on the site of departure (denoted $f$ for "from");

- capture probabilities $P$ that depend on the current site (denoted $c$ for "current"); and 


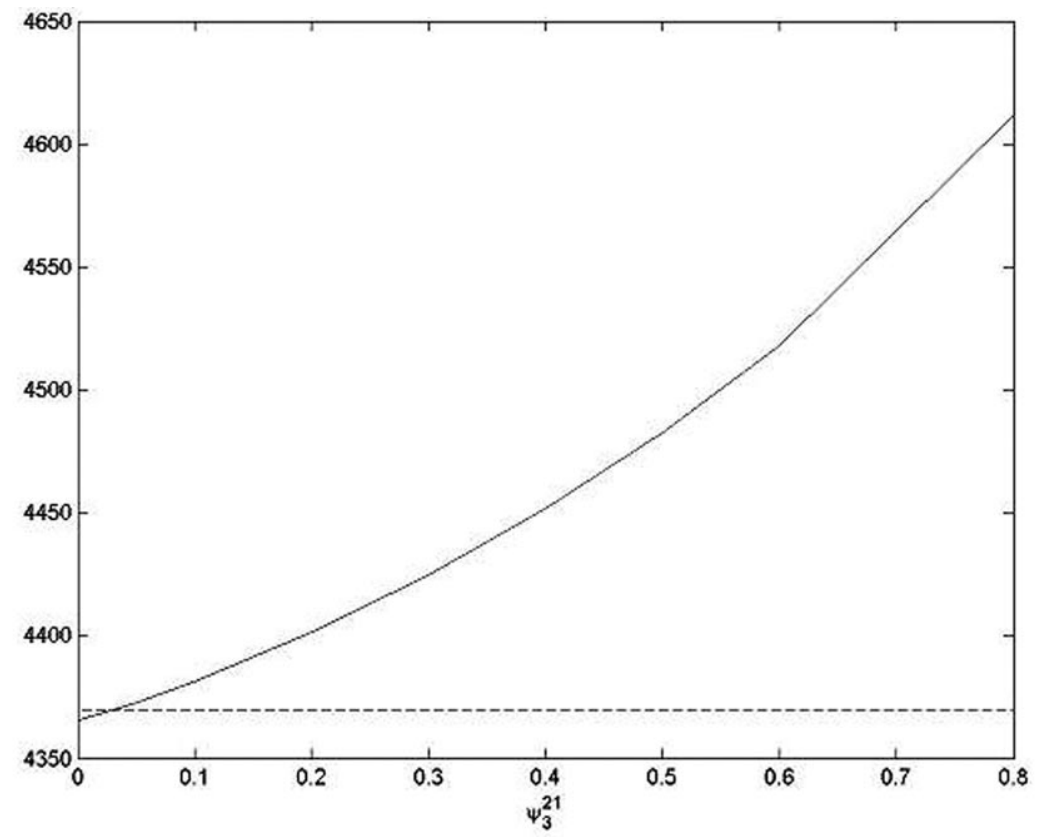

Figure 1. Graph of the profile deviance for the parameter $\psi_{3}^{B, N B}$ of the model $\left\{F_{f}, P_{c}, \Psi_{f . t o . t}\right\}$ (solid line) for the Sooty shearwater dataset (Scofield et al. 2001). The parameter $\psi_{3}^{B, N B}$ is estimated on the lower boundary, resulting in failure of usual maximum likelihood techniques to provide an estimate of variance. The profilelikelihood-based 95\% confidence interval is [0; .03]; it was obtained by a combination of the Venzon and Moolgavkar's algorithm and a cubic interpolation method (ten points were used for this example). The dashed line is the deviance at the MLE plus 3.84. Note that the deviance is -2log l, where l is the likelihood function.

- transitions probabilities $\Psi$ that depend on both site of departure and site of arrival (denoted $t o$ ) and time (denoted $t$ ); an interaction is indicated by the "dot" operator, for example, the interaction of sites and time in transition probabilities is expressed as $\Psi_{\text {f.to.t }}$.

In addition, for parameters, we used the same notation as in Scofield et al. (2001), where lower indices stand for time and upper indices for states. For example, the transition probability between occasions 3 and 4 from state breeder to state nonbreeder is denoted $\psi_{3}^{\mathrm{B}, \mathrm{NB}}$.

It has to be noted that the transition parameter $\psi_{3}^{\mathrm{B}, \mathrm{NB}}$ was estimated at the boundary of its domain, close to 0 (Figure 1). As a consequence, standard software such as MARK and MSURGE failed to compute Wald-type confidence intervals. In fact, usually the gradient at the MLE on a boundary is not zero and the matrix of variance-covariance is no longer reasonably estimated by the negative inverse of the Hessian, as assumed in the Wald approach. As with an estimate close to, but not on, the boundary, it is numerically difficult to obtain a good estimate of the Hessian. The profile-likelihood approach does not suffer from this problem. We therefore decided to use profile-likelihood intervals and to compare these with Wald-type intervals for different situations. 
The principle of the profile-likelihood approach to calculating confidence intervals is as follows. Let $\hat{\theta}$ be the MLE of a parameter vector $\theta \in \Theta \subseteq \Re^{p}$ and let $l(\bullet)$ be the log-likelihood function, then

$$
l(\hat{\theta})=\max _{\theta \in \Theta} l(\theta) .
$$

In the profile-likelihood approach, one considers $l(\theta)$ as a function of a single parameter of interest $\beta=\theta_{j}$, by treating the others as nuisance parameters and maximizing simultaneously over them. The profile likelihood for $\beta$ is thus defined as

$$
\bar{l}_{j}(\beta)=\max _{\theta \in \Theta_{j}(\beta)} l(\theta),
$$

where $\Theta_{j}(\beta)$ is a restriction of the parameter space $\Theta$ defined as $\Theta_{j}(\beta)=\left\{\theta \in \Theta \mid \theta_{j}=\beta\right\}$. A $100(1-2 \alpha) \%$ profile-likelihood confidence interval for $\theta_{j}$ is given by

$$
\left\{\beta \mid-2\left[\bar{l}_{j}(\beta)-l(\hat{\theta})\right] \leq q_{1}(1-\alpha)\right\},
$$

where $q_{1}(1-\alpha)$ is the $100(1-\alpha)$ th quantile of the $\chi^{2}$ distribution with one degree of freedom (see Cox and Hinkley 1974, p. 343). A technique commonly used to compute the confidence interval endpoints is derived directly from the definition (2.3): using, for example, the bisection method to find the zeroes of the function $\bar{l}_{j}(\beta)-l^{*}$, where $l^{*}$ is the constant $l^{*}=l(\hat{\theta})-.5 q_{1}(1-\alpha)$, the likelihood has to be optimized according to (2.2) at each step of the algorithm, resulting in two embedded levels of optimization. This task requires such a computational effort that its limited use can easily be understood.

\subsection{Venzon and Moolgavkar Algorithm}

Venzon and Moolgavkar (1988) proposed a faster algorithm for computing the confidence interval endpoints as solutions of the system of nonlinear equations

$$
F(\theta)=\left(\begin{array}{c}
l(\theta)-l^{*} \\
\frac{\partial l}{\partial \omega}(\theta)
\end{array}\right)=0,
$$

where $\omega=\left(\theta_{1}, \ldots, \theta_{j-1}, \theta_{j+1}, \ldots, \theta_{p}\right)^{\prime}$ is the parameter vector $\theta$ without its $j$ th coordinate. Because this is a single-step optimization problem, the computational burden is greatly reduced. In solving (2.4), Venzon and Moolgavkar (1988) proposed a two-fold modified Newton-Raphson algorithm (1) by providing as a starting value, the MLE $\hat{\theta}$ moved toward the left or right endpoint of the interval by an initial step $h$ and (2) by taking all the second derivatives of $l(\bullet)$ into account in the Newton-Raphson iterations in order to improve the rate of convergence toward interval endpoints.

\subsection{Finding the Best Starting Value}

The crucial point of this algorithm is a good starting value for the modified Newton algorithm, as it is well known to converge only locally. The first step proposed by Venzon 
and Moolgavkar (1988) implies that the gradient at the MLE is zero and that the likelihood is close to a quadratic function. When these assumptions are not met, $h$ may be too small or too large, resulting in nonconvergence of the algorithm. For this reason, we propose a modification to the computation of the starting value. The idea is to compute the profile likelihood at $n$ equally distributed values on $(0,1)$ and then use interpolation to find estimates of interval endpoints. The process used for computing the two interval endpoints is given in the following algorithm.

- Step 1: Form the vector of $n$ points on $(0,1)$ called $x=\left(x_{1}, \ldots, x_{n}\right)$, where $x_{i}=i /(n+1)$.

- Step 2: Calculate the vector $y=\left(y_{1}, \ldots, y_{n}\right)$ where $y_{i}-\bar{l}_{j}\left(x_{i}\right)=\max _{\theta / \theta_{j}=x_{i}} l(\theta)$.

- Step 3: Form the interpolation-function $w$ such that $w\left(x_{i}\right)=y_{i}, i=1, \ldots, n$. We chose $w$ to be a piecewise-cubic Hermite interpolation function.

- Step 4: Approximate the two closest points $x(1)$ and $x(2)$ to $\hat{\theta}_{j}$ such that for $k=$ $1,2, w(x(k))=l^{*}$, and the two vectors $z(k)=\arg \max _{\theta / \theta_{j}=x(i)} l(\theta)$. Form the two initial starting points $\left(z_{1}(i), \ldots, z_{j-1}(k), x(i), z_{j+1}(i), \ldots, z_{p}(i)\right)^{\prime}$ for $k=1,2$.

After a few attempts, we found that 10 points was a good choice in Step 1 so that this number was retained throughout the article. Note that Step 2 is time-consuming, but far less so than using a bootstrap approach. The algorithm described above works well and appears necessary in atypical cases (see Section 4). Nevertheless, it should be mentioned that the interpolation may make the calculations take longer in standard cases.

\subsection{Special Situations}

The procedure for the computation of profile-likelihood intervals relies on asymptotic normality of the MLE, as the deviance then has a $\chi^{2}$ distribution. When the parameter is at or close to a boundary, the asymptotic distribution of the deviance is known to be affected in a way that depends on whether other parameters are also at a boundary (Self and Liang 1987; Meeker and Escobar 1995). In developing our approach, we did not attempt to allow for this situation as multi-state capture-recapture parameters only lie on a boundary in very special cases. Survival probabilities will never be exactly 0 or 1 . Capture probabilities may be 0 when the field site is not visited, and may be 1 when all individuals are recaptured (Cam et al. 1998). In both these cases, we suggest setting the relevant parameter value to 0 or 1 and to then make inference conditional upon this value. When a parameter estimate is on a boundary (but the parameter intrinsically cannot be on such a boundary), we use our algorithm to compute only the other endpoint; see Section 4.

Extra-multinomial variation is another situation that requires consideration. When this is present, confidence intervals will be too narrow unless some adjustment is made to their calculation. Wald-type intervals are often adjusted by first estimating a variance-inflation or heterogeneity factor (Lebreton et al. 1992; Burnham et al. 1987), $\hat{c}$, as the ratio of the goodness-of-fit test for the model considered to its associated degrees of freedom df. The $(1-2 \alpha)$ Wald interval endpoints are then computed as $\hat{\theta} \pm t_{\mathrm{df}}(1-\alpha) \cdot \sqrt{\hat{c}} \cdot \hat{\sigma}_{\hat{\theta}}$, where 
Table 1. Coverage Rates for Wald and Profile-Likelihood 95\% Confidence Intervals. The model $\left\{F_{f}, P_{c}, \Psi_{f . t o}\right\}$ with two states and five capture occasions was considered. We used parameters $f^{1}=.6, f^{2}=.8, p^{1}=.4, p^{2}=.7, \psi^{12}=2 / 3, \psi^{21}=5 / 8$ to simulate 2,500 multistate capture-recapture history data sets. The states are labeled 1 and 2 .

\begin{tabular}{|c|c|c|c|c|c|c|c|}
\hline \multirow{2}{*}{$\begin{array}{c}\text { Number of newly } \\
\text { marked individuals }\end{array}$} & & \multicolumn{6}{|c|}{ Model $\left\{F_{f}, P_{c}, \Psi_{f . t o}\right\}$ parameters } \\
\hline & & $f^{1}$ & $f^{2}$ & $\Psi^{21}$ & $\Psi^{12}$ & $p^{1}$ & $p^{2}$ \\
\hline \multirow[t]{2}{*}{50} & Profile & .96 & .96 & .96 & .94 & .96 & .97 \\
\hline & Wald & .96 & .94 & .95 & .93 & .98 & .95 \\
\hline \multirow[t]{2}{*}{100} & Profile & .95 & .96 & .96 & .97 & .96 & .96 \\
\hline & Wald & .96 & .95 & .96 & .96 & .98 & .94 \\
\hline \multirow{2}{*}{500} & Profile & .95 & .95 & .95 & .95 & .95 & .95 \\
\hline & Wald & .95 & .95 & .95 & .95 & .95 & .95 \\
\hline
\end{tabular}

$t_{\mathrm{df}}(1-\alpha)$ is the $(1-\alpha)$ th quantile of the Student distribution with df degrees of freedom and $\hat{\sigma}_{\hat{\theta}}$ is the estimated standard error of $\theta$ (Lebreton et al. 1992). An analogous adjustment can be made for profile-likelihood intervals. This involves modifying (2.4) by replacing $l^{*}$ with $l(\hat{\theta})-.5 \cdot \hat{c} \cdot F_{1, \mathrm{df}}(1-\alpha)$ where $F_{1, \mathrm{df}}(1-\alpha)$ is the $(1-\alpha)$ th quantile of the Fisher distribution with 1 and df degrees of freedom; again, see Section 4.

\section{SIMULATION STUDY}

We considered three different situations, varying the number of encounter histories and the time- and state-dependency of the survival, capture and transition probabilities:

Scenario 1: Model $\left\{F_{f}, P_{c}, \Psi_{f . t o}\right\}$ with all parameters state-dependent only, $K=5$ and $a=2$ (Table 1).

Scenario 2: Model $\left\{F_{t}, P, \Psi_{\text {f.to }}\right\}$ with recapture and survival probabilities timedependent and transitions probabilities state-dependent, $K=3$ and $a=2$ (Table 2).

Table 2. Coverage Rates for Wald and Profile-Likelihood 95\% Confidence Intervals. The model $\left\{F_{t}, P_{t}, \Psi_{f . t o}\right\}$ with two states and three capture occasions was considered. We used actual parameters $f_{1}=.6, f_{2}=.8, p_{2}=.4, p_{3}=.7, \psi^{12}=2 / 3, \psi^{21}=5 / 8$ to simulate 2,500 multistate capture-recapture history datasets. The states are labeled 1 and 2 .

\begin{tabular}{cccccc}
\hline \hline \multirow{2}{*}{$\begin{array}{c}\text { Number of newly } \\
\text { marked individuals }\end{array}$} & & \multicolumn{4}{c}{ Model $\left\{F_{t}, P_{t}, \Psi_{f . t o}\right\}$ parameters } \\
\cline { 3 - 6 } & & $f_{1}$ & $p_{2}$ & $\psi^{21}$ & $\psi^{12}$ \\
\hline \multirow{2}{*}{50} & $\begin{array}{c}\text { Profile } \\
\text { Wald }\end{array}$ & .94 & .95 & .95 & .96 \\
& & .96 & .96 & .95 & .96 \\
& Profile & .95 & .95 & .95 & .95 \\
& Wald & .96 & .95 & .95 & .95 \\
& & & & .95 & .95 \\
& Profile & .95 & .95 & .95 & .95 \\
\hline
\end{tabular}


Table 3. Coverage Rates for Wald and Profile-Likelihood 95\% Confidence Intervals. The model $\left\{F, P, \Psi_{\text {f. to.t }}\right\}$ with two states and three capture occasions was considered. We used parameters $f=.6, p=.4, \psi_{1}^{21}=1 / 4, \psi_{1}^{12}=3 / 4, \psi_{2}^{21}=5 / 8, \psi_{2}^{12}=1 / 3$ to simulate 2,500 multistate capture-recapture history datasets. The states are labeled 1 and 2.

\begin{tabular}{cccccccc}
\hline \hline \multirow{2}{*}{$\begin{array}{c}\text { Number of newly } \\
\text { marked individuals }\end{array}$} & & \multicolumn{5}{c}{ Model $\left\{F, P, \Psi_{\text {f. to. } t\}}\right.$ parameters } \\
\cline { 3 - 8 } & & $f$ & $\psi_{1}^{21}$ & $\psi_{1}^{12}$ & $\psi_{2}^{21}$ & $\psi_{2}^{12}$ & $p$ \\
\hline \multirow{2}{*}{50} & Profile & .97 & .93 & .94 & .94 & .94 & .97 \\
& Wald & .93 & .96 & .97 & .96 & .97 & .98 \\
& & & & & & & \\
\multirow{3}{*}{100} & Profile & .96 & .94 & .94 & .94 & .95 & .96 \\
& Wald & .95 & .97 & .96 & .95 & .96 & .96 \\
& & & & & & .96 & .96 \\
500 & Profile & .95 & .95 & .95 & .94 & .95 & .94 \\
& Wald & .95 & .95 & .96 & .95 & .95 & .94 \\
\hline
\end{tabular}

Scenario 3: Model $\left\{F, P, \Psi_{\text {f.to.t }}\right\}$ with recapture and survival probabilities constant and transitions probabilities state- and time-dependent, $K=3$ and $a=2$ (Table 3 ).

In addition, following a referee's comment, we considered one more scenario. To obtain a situation comparable to the real case we examine in Section 4, we modified Scenario 1 in order to have a transition probability being near the boundary (see Table 4).

For each scenario, we simulated 2,500 capture-recapture history datasets using 50, 100, and 500 newly marked individuals for each occasion and state of release. For each set of data, profile-likelihood, and Wald-type 95\% confidence intervals were calculated, with the latter being calculated on the logit scale and then back-transformed. The estimated coverage rate was the proportion of the 2,500 intervals that contained the true parameter value. Because the MLE is only asymptotically unbiased, we calculated its mean square error (MSE). All programs were written in MATLAB (Version 6.5).

The results in Tables 1-3 show that, across all three scenarios, both the profile-likelihood and Wald-type intervals achieve the nominal coverage rate as long as the number of releases exceeds a hundred. Furthermore, when the number of releases is smaller, there appears to be no gain in using profile-likelihood rather than Wald-type intervals. In addition, a value near the boundary affects the corresponding parameter coverage rate and the other model parameters as well (Table 4).

Table 4. Coverage Rates for Wald and Profile-Likelihood 95\% Confidence Intervals. The model $\left\{F_{f}, P_{c}, \Psi_{\text {f. to }}\right\}$ with two states and five capture occasions was considered. We used parameters $f^{1}=.6, f^{2}=.8, p^{1}=.4, p^{2}=.7, \psi^{12}=2 / 3, \psi^{21}=.002$ to simulate 2,500 multistate capture-recapture history datasets. The states are labeled 1 and 2.

\begin{tabular}{cccccccc}
\hline \hline \multirow{2}{*}{$\begin{array}{c}\text { Number of newly } \\
\text { marked individuals }\end{array}$} & & \multicolumn{5}{c}{ Model $\left\{F_{f}, P_{c}, \Psi_{f . \text { to }}\right\}$ parameters } \\
\cline { 3 - 8 } 100 & & $f^{1}$ & $f^{2}$ & $\psi^{12}$ & $\psi^{21}$ & $p^{1}$ & $p^{2}$ \\
\hline \multirow{2}{*}{500} & Profile & .96 & .94 & .95 & .98 & .94 & .95 \\
& Wald & .92 & NA & .94 & NA & NA & NA \\
& & & & & & & \\
& Profile & .95 & .95 & .95 & .90 & .95 & .95 \\
& Wald & .94 & .95 & .95 & .84 & .96 & .93 \\
\hline
\end{tabular}




\section{EXAMPLE}

The sooty shearwaters dataset (Scofield et al. 2001) provides one illustration among others (Lebreton and Pradel 2002; Williams et al. 2002, pp. 454-468) of how multi-state capture-recapture models can be used to study trade-offs between costs of current reproduction on future survival and/or on future reproduction (Roff 1992; Stearns 1992). In what follows, we consider the simple model $\left\{F_{f}, P_{c}, \Psi_{f . t o . t}\right\}$ for illustration. We refer the reader to Scofield et al. (2001) for a complete treatment of the dataset. Following Gimenez, Choquet, and Lebreton (2003), we first checked that all parameters were separately estimable $\left\{F_{f}, P_{c}, \Psi_{\text {f.to.t }}\right\}$. Then, using the results of Pradel, Wintrebert, and Gimenez (2003), a goodness-of-fit test was performed for this model in order to estimate a variance inflation factor. We found that the level of extra-multinomial variation was high, with $\hat{c}=5.2$. We therefore calculated both raw and adjusted Wald-type and profile-likelihood intervals, as well as nonparametric bootstrap intervals (Table 5).

One MLE for the parameter $\psi_{3}^{\mathrm{B}, \mathrm{NB}}$ was on the boundary. Our algorithm leads to a $95 \%$ confidence interval for this parameter of $[.00 ; .03]$ (Figure 1). Both the profile-likelihood and bootstrap methods could be used for this parameter, unlike the Wald method. The unadjusted Wald-type and profile-likelihood intervals are very similar, while the bootstrap intervals appear to be more conservative (wider) in general, although in some cases the three methods gave similar results (except for parameter $\psi_{1}^{\mathrm{B}, \mathrm{NB}}$ ). Although the bootstrap approach automatically takes a part of the possible dependence and/or heterogeneity between individuals into account, the uncorrected Wald and profile-likelihood intervals are both highly affected in the same way. When adjusted for extra-binomial variation, both the Wald and profilelikelihood confidence intervals become wider than their bootstrap counterpart. These results seem to reflect the fact that (at least for a large "sample size") a nonparametric bootstrap could be expected to be an improvement over unadjusted theory-based methods, but that it would not necessarily allow for all of the overdispersion. Note that the profile-likelihood intervals were generally computed in approximately five minutes, while almost two and a half hours were required for the bootstrap intervals on a PC (Pentium II, 256Mo RAM, 1.2GHz CPU).

\section{DISCUSSION}

Profile-likelihood confidence intervals provide a compromise between Wald-type intervals and their bootstrap counterparts because (1) despite the high degree of nonlinearity in multi-state capture-recapture models, the coverage rates are comparable to nominal levels; (2) much less computation is required than for bootstrap intervals; and (3) using the algorithm presented here, the profile-likelihood approach can cope with boundary estimates.

Our simulation study suggests that profile-likelihood intervals do not perform better than Wald-type intervals when the sample size is small. It should be noted that we implicitly took the number of releases as our definition of the sample size (see, e.g., MacKenzie 2001). However, a high degree of data-sparseness can still occur with a large number of releases; for 
Table 5. Wald-Type, Nonparametric Bootstrap and Profile-Likelihood-Based Confidence-Intervals (Cl) for the Parameters of the Model $\left\{F_{f}, P_{c}, \Psi_{f \text {. to. } t}\right\}$ for the Sooty Shearwater Data (Scofield et al. 2001). Both the Wald-type and the profile-likelihood-based $\mathrm{Cl}$ are given under their unadjusted and adjusted for overdispersion form (see text).

\begin{tabular}{|c|c|c|c|c|}
\hline Parameters & $M L E$ & $\begin{array}{c}\text { Adjusted Wald Cl } \\
\text { Raw Wald Cl }\end{array}$ & Bootstrap Cl & $\begin{array}{c}\text { Adjusted Profile } \mathrm{Cl} \\
\text { Raw Profile } \mathrm{Cl}\end{array}$ \\
\hline$f^{\mathrm{NB}}$ & .81 & $\begin{array}{l}{[.71 ; .87]} \\
{[.77 ; .84]}\end{array}$ & {$[.75 ; .84]$} & $\begin{array}{l}{[.71 ; .89]} \\
{[.77 ; .84]}\end{array}$ \\
\hline$f^{\mathrm{B}}$ & .84 & $\begin{array}{l}{[.76 ; .89]} \\
{[.81 ; .86]}\end{array}$ & {$[.81 ; .86]$} & $\begin{array}{l}{[.75 ; .91]} \\
{[.81 ; .86]}\end{array}$ \\
\hline$p^{\mathrm{NB}}$ & .64 & $\begin{array}{l}{[.52 ; .75]} \\
{[.59 ; .69]}\end{array}$ & {$[.59 ; .69]$} & $\begin{array}{l}{[.51 ; .81]} \\
{[.59 ; .70]}\end{array}$ \\
\hline$p^{\mathrm{B}}$ & .53 & $\begin{array}{l}{[.42 ; .63]} \\
{[.48 ; .57]}\end{array}$ & {$[.42 ; .57]$} & $\begin{array}{l}{[.41 ; .65]} \\
{[.48 ; .57]}\end{array}$ \\
\hline$\psi_{1}^{\mathrm{B}, \mathrm{NB}}$ & .12 & $\begin{array}{l}{[.00 ; .81]} \\
{[.03 ; .38]}\end{array}$ & {$[.00 ; .29]$} & {$[.00 ; .78]$} \\
\hline$\psi_{1}^{\mathrm{NB}, \mathrm{B}}$ & .51 & $\begin{array}{l}{[.21 ; .80]} \\
{[.36 ; .65]}\end{array}$ & {$[.36 ; .65]$} & $\begin{array}{l}{[.16 ; .85]} \\
{[.36 ; .65]}\end{array}$ \\
\hline$\psi_{2}^{\mathrm{B}, \mathrm{NB}}$ & .02 & $\begin{array}{l}{[.00 ; .61]} \\
{[.00 ; .11]}\end{array}$ & {$[.00 ; .07]$} & $\begin{array}{l}{[.00 ; .24]} \\
{[.00 ; .07]}\end{array}$ \\
\hline$\psi_{2}^{\mathrm{NB}, \mathrm{B}}$ & .41 & $\begin{array}{l}{[.14 ; .74]} \\
{[.27 ; .56]}\end{array}$ & {$[.25 ; .69]$} & $\begin{array}{l}{[.08 ; .76]} \\
{[.26 ; .65]}\end{array}$ \\
\hline$\psi_{3}^{\mathrm{B}, \mathrm{NB}}$ & .00 & NA & {$[.00 ; .04]$} & $\begin{array}{l}{[.00 ; .16]} \\
{[.00 ; .03]}\end{array}$ \\
\hline$\psi_{3}^{\mathrm{NB}, \mathrm{B}}$ & .45 & $\begin{array}{l}{[.17 ; .76]} \\
{[.31 ; .59]}\end{array}$ & {$[.28 ; .59]$} & $\begin{array}{l}{[.12 ; .80]} \\
{[.30 ; .59]}\end{array}$ \\
\hline$\psi_{4}^{\mathrm{B}, \mathrm{NB}}$ & .01 & $\begin{array}{l}{[.00 ; .46]} \\
{[.00 ; .06]}\end{array}$ & {$[.00 ; .03]$} & $\begin{array}{l}{[.00 ; .14]} \\
{[.00 ; .04]}\end{array}$ \\
\hline$\psi_{3}^{\mathrm{NB}, \mathrm{B}}$ & .21 & $\begin{array}{l}{[.05 ; .56]} \\
{[.12 ; .35]}\end{array}$ & {$[.10 ; .36]$} & $\begin{array}{l}{[.03 ; .70]} \\
{[.12 ; .35]}\end{array}$ \\
\hline$\psi_{5}^{\mathrm{B}, \mathrm{NB}}$ & .24 & $\begin{array}{l}{[.12 ; .42]} \\
{[.17 ; .31]}\end{array}$ & {$[.16 ; .37]$} & $\begin{array}{l}{[.09 ; .43]} \\
{[.17 ; .30]}\end{array}$ \\
\hline$\psi_{5}^{\mathrm{NB}, \mathrm{B}}$ & .01 & $\begin{array}{l}{[.00 ; .45]} \\
{[.00 ; .06]}\end{array}$ & {$[.00 ; .05]$} & $\begin{array}{l}{[.00 ; .14]} \\
{[.00 ; .04]}\end{array}$ \\
\hline$\psi_{6}^{\mathrm{B}, \mathrm{NB}}$ & .68 & $\begin{array}{l}{[.46 ; .84]} \\
{[.59 ; .76]}\end{array}$ & {$[.53 ; .76]$} & $\begin{array}{l}{[.44 ; .87]} \\
{[.59 ; .76]}\end{array}$ \\
\hline$\psi_{6}^{\mathrm{NB}, \mathrm{B}}$ & .25 & $\begin{array}{l}{[.14 ; .41]} \\
{[.19 ; .32]}\end{array}$ & {$[.19 ; .36]$} & $\begin{array}{l}{[.12 ; .43]} \\
{[.19 ; .31]}\end{array}$ \\
\hline
\end{tabular}

example, when capture probabilities are high, resulting in many empty cells in the $m$-array. In such cases, classical theory does not hold (e.g., Bishop, Fienberg, and Holland 1975, pp. 410-413). An extensive simulation study would be needed to explore and compare the effect of sparse data on both profile-likelihood and Wald-type interval coverage rates; this is the focus of current research. Note that CJS-type models are special cases of multi-state models, and so our conclusions should also apply generally to those cases.

All the methods we have considered here assume that the likelihood is bell-shaped, at least locally, near its maximum. This may not always be the case, especially with multi-state models which are known to produce likelihoods with local maxima (Lebreton and Pradel 2002). The situation shown in Figure 2 might then arise, where the confidence region is the union of two distinct intervals. The Wald approach cannot deal with this type of situation, whereas, in principle, both the bootstrap and profile-likelihood approaches can. In practice, 


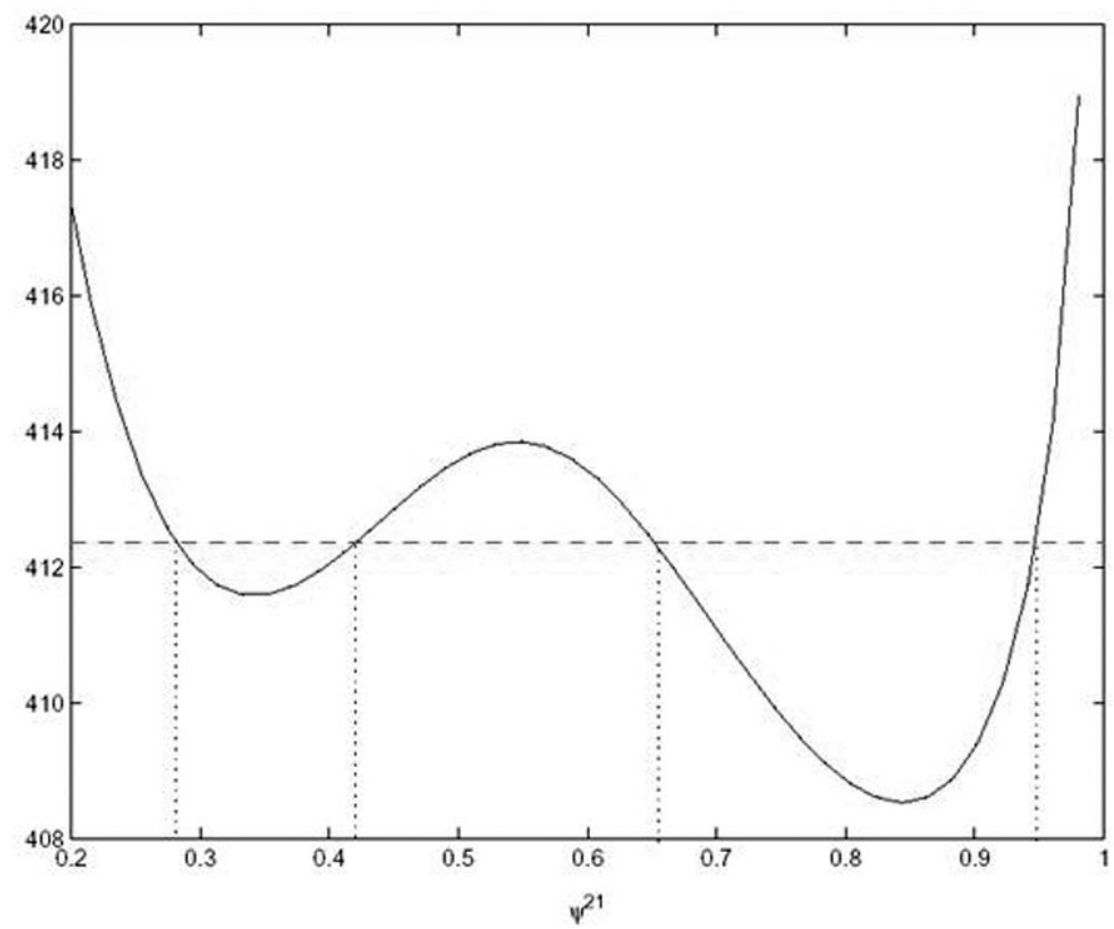

Figure 2. Graph of the profile deviance in presence of a local minimum. We fit the model $\left\{F, P, \Psi_{\text {f.to.t }}\right\}$ to the following artificial dataset with 7 capture occasions and 2 states (the number of times that the encounter history is observed is between parenthesis): 2021202 (4); 2020201 (4); 2020202 (4); 2201021 (4); 1110101 (4); 1010101 (4); 1010102 (4); 2102011 (4) (Dupuis, pers. comm. and Gimenez 2003). The dashed line is the deviance at the MLE plus 3.84. The 95\% profile confidence interval for $\psi^{21}$ is a reunion of both the two intervals [.28; .42] and [.65; .95]. A similar plot can be obtained for $\psi^{12}$ with $[.20 ; .32] \cup[.45 ; .74]$ as a $95 \%$ profile confidence interval. Note that the deviance is $-2 \log l$, where $l$ is the likelihood function.

the algorithm we have presented here computes the deviance at several points and it is possible to check the form of the likelihood function near the MLE. A more systematic search might be considered in order to look for local minima. In general it is prudent to examine profile likelihoods rather than simply calculate point estimates (Box and Jenkins 1976, pp. 224-226; Meeker and Escobar 1995; Lebreton and Pradel 2002).

The algorithm presented here is implemented in software M-SURGE (Choquet et al. 2003), a program specifically written to deal with and take advantage of recent developments in multi-state capture-recapture models. It is freely available through the Internet at ftp: //ftp.cefe.cnrs-mop.fr/biom/Soft-CR/.

[Received February 2004. Revised February 2005.]

\section{REFERENCES}


Arnason, A. N. (1972), "Parameter Estimates from Mark-Recapture Experiments on Two Populations Subject to Migration and Death," Researches on Population Ecology, 13, 97-113.

(1973), "The Estimation of Population Size, Migration Rates and Survival in a Stratified Population," Research on Population Ecology, 15, 1-8.

Bates, D. M., and Watts, D. G. (1980), "Relative Curvature Measures of Nonlinearity," Journal of the Royal Statistical Society, Ser. B, 42, 1-25.

Beale, E. M. L. (1960), “Confidence Regions in Nonlinear Estimation," Journal of the Royal Statistical Society, Ser. B, 22, 41-88.

Bishop, Y. M., Fienberg, S. E., and Holland, P. (1975), Discrete Multivariate Analysis: Theory and Practice, Cambridge, MA: MIT Press.

Box, J. E. P., and Jenkins, G. W. (1976), Time Series Analysis: Forecasting and Control, San Francisco: Holden-Day.

Brownie, C., Hines, J. E., Nichols, J. D., Pollock, K. H., and Hestbeck, J. B. (1993), "Capture-Recapture Studies for Multiple Strata Including non-Markovian Transitions," Biometrics, 49, 1173-1187.

Buckland, S. T. (1980), “A Modified Analysis of the Jolly-Seber Capture-Recapture Model," Biometrics, 36, 419-435.

Buckland, S. T., and Garthwaite, P. H. (1991), "Quantifying Precision of Mark-Recapture Estimates Using the Bootstrap and Related Methods," Biometrics, 47, 255-268.

Burnham, K. P., Anderson, D. R., White, G. C. Brownie, C., and Pollock, K. H. (1987), "Design and Analysis Methods for Fish Survival Experiments Based on Release-Recapturel," Bethesda, MD: American Fisheries Society.

Cam, E., Hines, J. E., Monnat, J.-Y., Nichols, J. D., and Danchin, E. (1998), “Are Adult Non-breeders Prudent Parents? The Kittiwake Model," Ecology, 79, 2917-2930.

Choquet, R., Reboulet, A. M., Pradel, R., Gimenez, O., and Lebreton, J.-D. (2003), User's Manual for M-SURGE 1.0, Montpellier, France: CEFE/CNRS.

Clobert, J. (1995), “Capture-Recapture and Evolutionary Ecology: A Difficult Wedding?” Journal of Applied Statistics, 22, 989-1008.

Cook, R. D., and Weisberg, S. (1990), "Confidence Curves in Nonlinear Regression," Journal of the American Statistical Association, 85, 544-551.

Cormack, R. M. (1964), "Estimates of Survival From the Sighting of Marked Animals," Biometrika, 51, 429-438. (1992), "Interval Estimation for Mark-Recapture Studies of Closed Populations," Biometrics, 48, 567-576.

Cox, A. Z., and Hinkley, A. Z. (1974), Theoretical Statistics, London: Chapman and Hall.

Davison, A. C., and Hinkley, D. V. (1997), Bootstrap Methods and Their Applications, Cambridge: University Press.

Donaldson, J. R., and Schnabel, B. (1987), "Computational Experiences for Confidence Regions and Confidence Intervals for Nonlinear Least Squares," Technometrics, 29, 67-82.

Efron, B., and Tibshirani, R. J. (1993), An Introduction to the Bootstrap, New York: Chapman and Hall.

Evans, M. A., Kim, H.-M., and O’Brien, T. E. (1996), "An Application of Profile-Likelihood Based Confidence Interval to Capture-Recapture Estimators," Journal of Agricultural, Biological, and Environmental Statistics, $1,131-140$.

Garthwaite, P. H., and Buckland, S. T. (1992), "Generating Monte Carlo Confidence Intervals by the RobbinsMonro Process," Applied Statistics, 41, 159-171.

Gimenez, O. (2003), "Estimation et Tests d'Adéquation pour les Modéles de Capture-Recapture Multiétats," unpublished $\mathrm{PhD}$ thesis, University of Montpellier II.

Gimenez, O., Choquet, R., and Lebreton, J. D. (2003), "Parameter Redundancy in Multi-state Capture-Recapture Models," Biometrical Journal, 45, 704-722.

Hines, J. E. (1994), MSSURVIV User's Manual, Laurel, MD: National Biological Survey.

Hudson, D. J. (1971), "Interval Estimation from the Likelihood Function," Journal of the Royal Statistical Society, Ser. B, 33, 256-262.

Huggins, R. M. (1989), “On the Statistical Analysis of Capture Experiments,” Biometrika, 76, 133-140. 
Jolly, G. M. (1965), "Explicit Estimates From Capture-Recapture Data with Both Death and ImmigrationStochastic Model," Biometrika, 52, 225-247.

Lebreton, J.-D. (1995), “The Future of Population Dynamic Studies Using Marked Animals: A Statistician's Perspective," Journal of Applied Statistics, 22, 1009-1030.

Lebreton, J.-D., Almeras, T., and Pradel, R. (1999), “Competing Events, Mixtures of Information and Multistratum Recapture Models," Bird Study, 46 (suppl.), S39-46.

Lebreton, J.-D., Burnham, K. P., Clobert, J. and Anderson, D. R. (1992), "Modeling Survival and Testing Biological Hypotheses Using Marked Animals: A Unified Approach With Case Studies," Ecological Monographs, 62, $67-118$.

Lebreton, J. D., Hines, J. E., Pradel, R., Nichols, J. D., and Spendelow, J. A. (2003), "Estimation by CaptureRecapture of Recruitment and Dispersal Over Several Sites," Oikos, 101, 253-264.

Lebreton, J. D., and Pradel, R. (2002), "Multi-State Recapture Models: Modelling Incomplete Individual Histories," Journal of Applied Statistics, 29, 353-369.

Lloyd, C. J. (1995), “Accurate Confidence Intervals from Recapture Experiments," Communications in StatisticsTheory and Methods, 24, 1235-1253.

MacKenzie, D. (2001), “Assessing Mark-Recapture Data with Computer Intensive Statistics,” PhD thesis, Otago: University of Otago.

Meeker, W. Q., and Escobar, L. A. (1995), “Teaching About Approximate Confidence Regions Based on Maximum Likelihood Estimation," The American Statistician, 49, 48-53.

Morgan, B. J. T., and Freeman, S. N. (1989), "A Model With First-Year Variation for Ring-Recovery Data," Biometrics 45, 1087-1101.

Nichols, J. D., and Kendall, W. L. (1995), “The Use of Multi-state Capture-Recapture Models to Address Questions in Evolutionary Ecology," Journal of Applied Statistics, 22.

Pradel, R., Wintrebert, C., and Gimenez, O. (2003), "A Proposal for a Goodness-of-Fit Test to the Arnason-Schwarz Multisite Capture-Recapture Model," Biometrics, 59, 43-53.

Ratkowsky, D. A. (1988), Handbook of Nonlinear Regression Models, New York: Marcel Dekker.

Roff, D. A. (1992), The Evolution of Life Histories-Theory and Analysis, New York: Chapman and Hall.

Schwarz, C. J., Schweigert, J. F., and Arnason, A. N. (1993), "Estimating Migration Rates Using Tag-Recovery Data," Biometrics, 49, 177-193.

Scofield, R. P., Fletcher, D. J., and Robertson, C. J. R. (2001), "Titi (Sooty Shearwaters) on Whero Island: Analysis of Historic Data Using Modern Techniques," Journal of Agricultural, Biological, and Environmental Statistics, 6, 268-280.

Seber, G. A. F. (1965), "A Note on the Multiple-Recapture Census," Biometrika, 52, 249-259.

Seber, G. A. F., and Schwarz, C. J. (2002), "Capture-Recapture: Before and After EURING 2000," Journal of Applied Statistics, 29, 5-18.

Self, S. G., and Liang, K. Y. (1987), "Asymptotic Properties of Maximum Likelihood Estimators and Likelihood Ratio Tests Under Nonstandard Conditions," Journal of the American Statistical Association, 398, 605-610.

Stearns, S. C. (1992), The Evolution of Life Histories, Oxford: Oxford University Press.

Venzon, D. J., and Moolgavkar, S. H. (1988), "A Method for Computing Profile-Likelihood-Based Confidence Intervals," Applied Statistics, 37, 87-94.

Viallefont, A., and Lebreton, J.-D. (1993), "Estimation des taux de Survie et de Migration par CaptureRecapture dans des Populations Animales Stratifiées Spatialement," Biométrie et Analyse des Données Spatio-Temporelles, Société Française de Biométrie, 12.

White, G. C., Anderson, D. R., Burnham, K. P., and Otist, D. L. (1982), Capture-Recapture and Removal Methods for Sampling Closed Populations, Los Alamos National Laboratory.

White, G. C., and Burnham, K. P. (1999), "Program MARK: Survival Estimation from Populations of Marked Animals," Bird Study 46 (suppl.), 120-139.

Williams, B. K., Nichols, J. D., and Conroy, M. J. (2002), Analysis and Management of Animal Populations, San Diego, CA: Academic Press. 